\title{
Relationship Between Salivary Melatonin and Severity of Periodontal Disease
}

\author{
Antonio Cutando, * Pablo Galindo, ${ }^{\dagger}$ Gerardo Gómez-Moreno, * Carlos Arana, * Jorge Bolaños, ${ }^{\ddagger}$ \\ Darío Acuña-Castroviejo, $§$ and Hom-Lay Wangl|
}

Background: Melatonin possesses antioxidant, free-radical scavenging, and immunoenhancing properties that promote fibroblast activity and bone regeneration. The aim of this study was to examine the possible links between salivary melatonin levels and the severity of periodontal disease using the community periodontal index (CPI).

Methods: Thirty-seven patients with different degrees of periodontal disease were studied. Salivary and plasma melatonin levels (by radioimmunoassay), salivary/plasma melatonin ratio, and CPI status were collected for each patient. The Spearman correlation coefficient was used to analyze relationships among variables.

Results: Data showed a significant correlation between CPI and salivary/plasma melatonin ratios. When saliva volume was controlled for, a significant correlation $(P<0.05)$ was found between lower salivary melatonin and a worse CPI. This finding suggests that melatonin may act as a protector against free radicals produced by inflammatory periodontal diseases.

Conclusions: Salivary melatonin levels varied according to the degree of periodontal disease. As the degree of periodontal disease increased, the salivary melatonin level decreased, indicating that melatonin may act to protect the body from external bacterial insults. Therefore, melatonin may be potentially valuable in the treatment of periodontal diseases, although further research is required to validate this hypothesis. J Periodontol 2006; 77:1533-1538.

\section{KEY WORDS}

Melatonin; periodontal diseases; periodontal index; plasma; saliva.

\footnotetext{
* Department of Special Care in Dentistry, School of Dentistry, University of Granada, Granada, Spain.

$\uparrow$ Department of Oral Surgery, School of Dentistry, University of Granada.

\# Statistics and Operational Research Department, Faculty of Library Science and

Documentation, University of Granada

$\S$ Department of Physiology, Institute of Biotechnology, University of Granada.

|| Department of Periodontics and Oral Medicine, School of Dentistry, University of Michigan, Ann Arbor, MI.
}

$M$ elatonin is an indoleamine produced in various parts of the body, mainly in the pineal gland. ${ }^{1}$ This gland produces melatonin in a circadian manner, synchronizing a number of biologic processes in a 24-hour, day-night rhythm. Melatonin has strong antioxidant effects ${ }^{2}$ that can protect cells against inflammatory processes and oxidative damage. ${ }^{3-5}$ These inflammatory processes include the development of certain cancers, ionizing radiations, metabolism alterations, and inflammatory responses. ${ }^{6}$ Melatonin also plays an immunomodulatory role $^{7}$ via the secretion of interleukin-2 (IL-2) and interferon- $\alpha$ (INF- $\alpha$ ) and the consequent activation of CD4+ lymphocytes. ${ }^{8}$ Furthermore, recent studies reported that melatonin may stimulate the proliferation and synthesis of type I collagen and promote bone formation. ${ }^{9,10}$

After release of melatonin into the blood stream, it diffuses into the saliva. The proportion of plasma melatonin passing into the mouth via salivary glands appears to be relatively stable, ranging from $24 \%$ to $33 \% .{ }^{11}$ It is widely agreed that $70 \%$ of plasma melatonin is albumin bound. Because only the free melatonin in plasma is thought to be present in saliva, salivary melatonin levels reflect the proportion of free-circulating melatonin. ${ }^{12}$ Hence, the measurement of salivary melatonin levels represents an indirect, non-invasive method for the assessment of plasma melatonin levels. 
Among other functions, saliva is important for maintaining oral health status: it lubricates the mouth; protects against harmful substances in the environment; modulates oral flora; initiates digestion; and contributes to tasting, swallowing, and speaking. It also has various immunological functions because it contains immunoglobulin A (IgA) antibodies and other antimicrobial molecules such as lysozyme, lactoferrin, and peroxidase. Saliva also protects teeth by neutralizing acids produced by carbohydrate fermentation.

A reduction in the salivary secretion rate depends on a number of factors. Unstimulated salivary rates below 0.1 to $0.2 \mathrm{ml} /$ minute and stimulated rates below 0.5 to $0.7 \mathrm{ml} /$ minute are considered abnormal. Factors that reduce salivary flow include certain drugs (e.g., antidepressants, antihypertensives, and diuretics), systemic diseases (e.g., diabetes and hypertension), autoimmune diseases (e.g., Sjögren's syndrome), and others (e.g., postradiotherapy glandular atrophy and aging). The xerostomia produced by these factors gives rise to morphological and pathophysiological changes in the oral mucosa and may cause various oral diseases. ${ }^{13}$

Given the properties of melatonin and its presence in the mouth via saliva, ${ }^{14}$ its effect on oral health, especially periodontal health, warrants further investigation. Our group previously examined the relationship between salivary melatonin and oral health in diabetic patients. ${ }^{15}$ The measurement of melatonin levels in saliva and plasma should enhance our understanding of this interaction. Therefore, the aim of this study was to examine the relationship between the level of salivary melatonin and the community periodontal index (CPI) score ${ }^{16}$ used to assess periodontal disease status.

\section{MATERIALS AND METHODS}

Thirty-seven periodontitis patients, 21 females and 16 males, with a mean age of $55.1 \pm 0.46$ years (range: 18 to 65 years) were recruited at the University of Granada. Exclusion criteria were as follows: presence of a systemic neurological disorder (e.g., epilepsy or schizophrenia), presence of disease with possible effects on the immune system (e.g., chronic infections or cancer), and treatment with any drug that might alter melatonin levels. The study was approved by the University Ethics Committee and was performed in accordance with the Code of Ethics of the World Medical Association. Written informed consent was obtained from all patients.

All study patients underwent an oral examination, including medical, dental, and caries assessments, and CPI scores were collected for each patient. The same dentist performed all examinations. A concordant diagnostic analysis was performed on 11 ran- domly selected patients by a second examiner, yielding an interobserver concordance coefficient of $81 \%$ for CPI assessments. A physiologist masked to the oral status of patients measured the plasma and salivary melatonin levels of each patient.

\section{Measurement of Salivary Melatonin Levels}

Participants were asked to refrain from eating after midnight on the day of the saliva sample collection, which was carried out around 8:30 am. To stimulate saliva flow, the patients were asked to chew a piece of paraffin wax for 7 minutes. The saliva produced in the first 2 minutes was discarded, and only the saliva generated in the remaining 5 minutes was collected and centrifuged at 3,000 rpm for 20 minutes. The supernatant was collected and frozen at $-20^{\circ} \mathrm{C}$ until analysis. The amount of melatonin in saliva was determined by a specific commercial radioimmunoassay. . ${ }^{\text {I17 }}$ The intra- and interassay coefficients of variation shown by the quality control of this method were $12.9 \%$ and $7.2 \%$, respectively. The recovery of the added melatonin was $80 \%$, and the assay sensitivity was $0.04 \mathrm{nmol} / \mathrm{l}$.

\section{Measurement of Serum Melatonin Levels}

A 10-ml blood sample was also drawn from the antecubital vein of each patient. This was done at the same time that the saliva sample was collected. Blood samples were kept at room temperature for 2 hours and centrifuged at 3,000 rpm for 10 minutes, and extracted serum was frozen at $-20^{\circ} \mathrm{C}$.

A commercial radioimmunoassay was employed to determine the amount of serum melatonin. ${ }^{\# 18}$ The intra- and interassay coefficients of variation displayed by the quality control of this method were $11.3 \%$ and $6.3 \%$, respectively. The recovery of the added melatonin was $84.4 \%$, and assay sensitivity was $0.02 \mathrm{nmol} / \mathrm{l}$.

\section{Assessment of Periodontal Disease Severity}

Periodontal disease severity was evaluated using the CPI. The CPI is determined by dividing the mouth into sextants, each of which contains index teeth. The sextants are teeth \# 1 through \#5, \#6 through \#11, \#12 through \# 16, \#17 through \#21, \#22 through \#27, and \#28 through \#31. The periodontal examination was carried out using a probe recommended by the World Health Organization (WHO) with two color markings at 3.5 and $5.5 \mathrm{~mm}$ to record the degree of periodontal bone loss. CPI codes used for assessing periodontal status are as follows: code $0=$ healthy; code $1=$ bleeding observed, directly or by using a mirror, after sensing; code 2 = calculus felt during probing, but all of the black band on the probe was visible; code $3=a$ pocket of 4 to $5 \mathrm{~mm}$ (the gingival margin within the

II IBL Hamburg, Hamburg, Germany.

\# DVD Biochemie, Marburg, Germany. 
black band on the probe); code $4=$ a pocket $\geq 6 \mathrm{~mm}$ (the black band on the probe was not visible). ${ }^{16}$

\section{Statistical Analysis}

Means and standard deviations were calculated for the following study variables: patient age, saliva volume, CPI, salivary melatonin, plasma melatonin, and salivary/plasma melatonin ratio. A multiple regression analysis was performed to include all variables. The statistical significance of associations among variables was determined by using the Spearman correlation coefficient.

\section{RESULTS}

Means and standard deviations of measurement variables were as follows: saliva volume: $2.99 \pm 0.49 \mathrm{ml}$; plasma melatonin level: $8.80 \pm 1.44 \mathrm{pg} / \mathrm{ml}$; salivary melatonin level: $2.65 \pm 0.43 \mathrm{pg} / \mathrm{ml}$; salivary/plasma melatonin ratio: $0.31 \pm 0.05$; and CPI: $2.57 \pm 0.42$.

Results are shown in Table 1. Older patients had lower saliva volumes $(\rho=-0.537 ; P<0.01)$, higher CPI scores $(\rho=0.516 ; P<0.01)$, and lower salivary/ plasma melatonin ratios $(\rho=-0.035 ; P<0.05)$ compared to younger patients. Patients with a CPI score of 2 had lower levels of plasma and salivary melatonin. When CPI scores were above 2, corresponding to a higher mean age, an increase in plasma and salivary melatonin levels was observed.

A multiple regression analysis showed that age, saliva volume, and gingival index were significantly associated with the CPI. Gender and smoking had no correlation with melatonin levels. Age and saliva volume alone were also included because they were the only variables significantly related to melatonin level.

A larger saliva volume was associated with a higher salivary/plasma melatonin ratio $(\rho=0.369 ; P<0.05)$, even after controlling for the saliva volume $(\rho=$
$-0.391 ; P<0.05)$ (Fig. 1). Patients with higher salivary and plasma melatonin ratios had lower CPI scores $(\rho=-0.390 ; P<0.05)$ (Table 2$)$.

\section{DISCUSSION}

Data from this study indicated that the amount of serum melatonin secreted by salivary glands varied according to the severity of the periodontal disease: the higher the CPI score (worsening of periodontal disease), the lower the salivary melatonin level and salivary/plasma melatonin ratio. This finding suggests that the melatonin may possess the ability to fight against infection and inflammation, probably due to its antioxidant, anti-aging, and immunoenhancing action. $., 9,19-22$

Periodontal disease is well known to be associated with an inflammation of the periodontium that destroys periodontal ligament and alveolar bone by resorptive processes. These processes mainly involve osteoclasts, which are mediated by cytokines and local factors released by neighboring defensive cells in response to established bacterial aggression. Melatonin has a critical function in the regulation of proteins implicated as mediators of these processes. The receptor activator of nuclear factor-kappa B ligand (RANKL) is a highly important protein in osteoclastic differentiation and proliferation. ${ }^{23}$ Another protein, osteoprotegerin (OPG), interferes with its biologic potential. Liu et al. ${ }^{24}$ demonstrated that these proteins play a critical role in the development of periodontal disease, with periodontal bone destruction produced by the upregulation of RANKL with downregulation of OPG. Melatonin can modulate these events because it is closely related to orchestration of the molecular triad OPG/RANK/RANKL. ${ }^{25}$ Thus, treatment with melatonin, besides stimulating the proliferation, differentiation, and activity of osteoblasts, ${ }^{9}$

\section{Table I.}

\section{Non-Parametric Correlations Among Measurement Variables}

\begin{tabular}{lcccccc}
\hline Variable & Age & $\begin{array}{c}\text { Saliva } \\
\text { Volume }\end{array}$ & $\begin{array}{c}\text { Plasma } \\
\text { Melatonin }\end{array}$ & $\begin{array}{c}\text { Salivary } \\
\text { Melatonin }\end{array}$ & $\begin{array}{c}\text { Salivary/Plasma } \\
\text { Melatonin Ratio }\end{array}$ & CPI \\
\hline Age & 1.000 & $-0.537^{\dagger}$ & 0.018 & -0.048 & $-0.353^{*}$ & $0.516^{\dagger}$ \\
Saliva volume & $-0.537^{\dagger}$ & 1.000 & $0.395^{*}$ & $0.389^{*}$ & $0.369^{*}$ & $-0.295^{\dagger}$ \\
Plasma melatonin & 0.018 & $0.395^{*}$ & 1.000 & $0.981^{\dagger}$ & 0.118 & 0.054 \\
Salivary melatonin & -0.048 & $0.389^{*}$ & $0.981^{\dagger}$ & 1.000 & 0.218 & -0.130 \\
Salivary/plasma melatonin ratio & $-0.353^{*}$ & $0.369^{*}$ & 0.118 & 0.218 & 1.000 & $-0.390^{*}$ \\
CPI & $0.516^{\dagger}$ & $-0.295^{\dagger}$ & 0.054 & -0.130 & $-0.390^{*}$ & 1.000 \\
\hline
\end{tabular}

Spearman correlation analysis $(\mathrm{N}=37)$.

* $P<0.05$.

$\dagger P<0.01$. 
Table 2.

\section{Correlation Analyses Among Variables, Controlling for Saliva Volume}

\begin{tabular}{lccccc}
\hline Variable & Age & Plasma Melatonin & Salivary Melatonin & Salivary/Plasma Melatonin Ratio & CPI \\
\hline Age & 1.000 & 0.290 & 0.230 & $0.395^{*}$ & -0.348 \\
Plasma melatonin & 0.290 & 1.000 & $0.985^{\dagger}$ & 0.148 & -0.100 \\
Salivary melatonin & 0.230 & $0.985^{\dagger}$ & 1.000 & 0.073 & 0.063 \\
Salivary/plasma melatonin ratio & 0.395 & 0.147 & 0.734 & 1.000 & $-0.391^{*}$ \\
CPI & -0.348 & -0.100 & 0.063 & $-0.391 *$ & 1.000 \\
\hline
\end{tabular}

Spearman correlation analysis $(\mathrm{N}=37)$.

* $P<0.05$.

$\dagger P<0.01$.

reduces the expression of RANKL in vivo and increases the production of OPG by osteoblasts. ${ }^{10}$ In this way, melatonin inhibits osteoclastogenesis mediated by an imbalance in favor of RANKL, as occurs in periodontal disease.

Prostaglandin $\mathrm{E}_{2}\left(\mathrm{PGE}_{2}\right)$ has also been implicated in osteoclastic activity in periodontal disease, largely in relation to periodontal bone loss. ${ }^{26}$ Melatonin can also modulate the development of periodontal disease in this context because it can act on $\mathrm{PGE}_{2}$, thereby inhibiting the differentiation of osteoclasts induced by

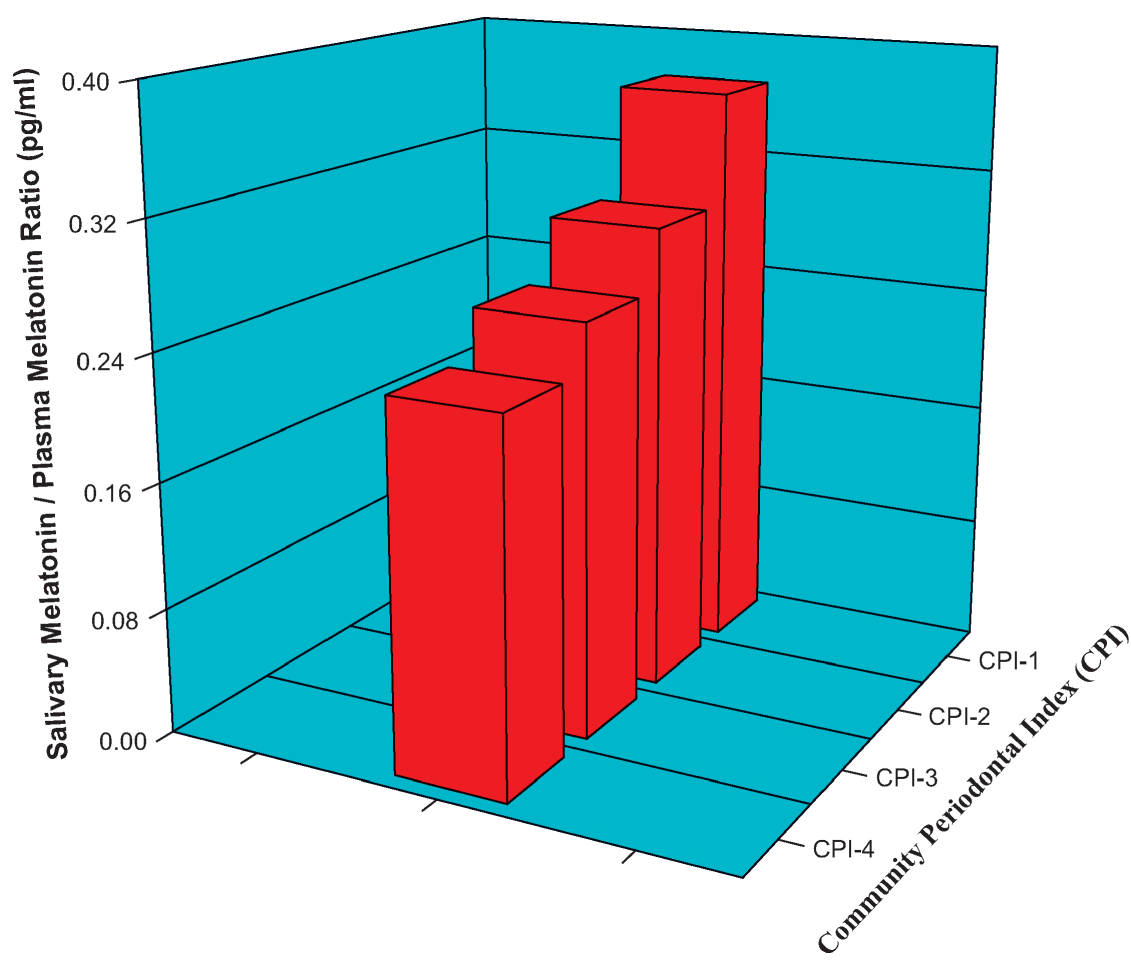

Figure I.

Relationship between salivary/plasma melatonin ratio and CPI, controlling for saliva volume. cell-to-cell contact between osteoblasts and osteoclasts. ${ }^{27}$ Moreover, melatonin can modulate parathormone (PTH), ${ }^{28} 1 \alpha 25\left(\mathrm{OH}_{2}\right) \mathrm{D}_{3}$, IL-1 $\beta$, and IL-6, ${ }^{25}$ all proteins that regulate the resorption process in periodontal disease and interact with other biologic agents such as calcitonin ${ }^{29}$ or IL-2. ${ }^{30}$ Therefore, melatonin may inhibit bone resorption ${ }^{31}$ or promote bone-marrow cell differentiation.

It is important to note that the stimulation of osteoblastic differentiation by melatonin may influence bone formation through the induction of bone sialoprotein $^{32}$ and alkaline phosphatase and osteocalcin ${ }^{22}$ that are essential proteins for bone maturation. Hence, melatonin may play a major role in the regulation of the bone resorptionformation balance in favor of bone formation.

Melatonin activates several elements of the immune system that reduce tissue destruction during the inflammatory response, either directly by free radical scavenging or indirectly by modulating the action of agents such as cytokines and adhesion molecules, which contribute to the advance of cell damage. ${ }^{6}$ In summary, melatonin may play an important role in maintaining periodontal health.

The finding of a significant association among patient age, decreased saliva volume, and poor periodontal status (high CPI) supports the positive correlation between age-related xerostomia and poor periodontal status previously reported by our group ${ }^{15}$ and other authors. ${ }^{33}$ Patients with a salivary gland dysfunction 
always had a low salivary melatonin level, which is known to result in decreased antioxidant defenses against the free radicals released during oral inflammation/infection. ${ }^{15}$ Battino et al. $^{33}$ observed a decrease in the salivary/serum melatonin ratio with higher age and concluded that melatonin levels in the oral cavity were reduced by a lower clearance of serum melatonin by salivary glands. It has been reported that the salivary infiltration rate in many elderly people is not maintained within $24 \%$ to $33 \%$ of the normal range. ${ }^{11}$ In the present study, normal salivary melatonin levels were observed in patients when the saliva volume was also within the normal range. This observation suggests that salivary glands may control the amount of melatonin passing from the blood into the oral cavity. This mechanism is of great importance because melatonin is not produced in the mouth, and only serum melatonin can reach it.

With the above background, it can be speculated that there is a negative association between the salivary melatonin level and periodontal disease severity. Consequently, the reduction in saliva production with higher age and the decreased melatonin production in older adults reflected in low salivary melatonin levels predispose these individuals to an increased risk of developing oral and periodontal disease.

To our knowledge, this is one of the first attempts to examine the influence of salivary melatonin upon periodontal disease. Data from this study suggest that melatonin may be able to protect the oral cavity against free radicals produced by inflammatory diseases. Further research with adequate sample power is required to fully elucidate the relationship between serum melatonin and salivary melatonin and its influence on periodontal health.

\section{ACKNOWLEDGMENTS}

This work was supported by the Carlos III Health Institute, Madrid, Spain (grant FIS PI041610); the Andalusia Regional Education Council, Seville, Spain; and Implant Microdent System, Barcelona, Spain (grants CTS-263 and CTS-101).

\section{REFERENCES}

1. Reiter RJ. Pineal melatonin: Cell biology of its synthesis and of its physiological interactions. Endocr Rev 1991;12:151-180.

2. Reiter RJ, Tan DX, Acuña-Castroviejo D, Burkhardt S, Karbownik M. Melatonin: Mechanics and actions as an antioxidant. Curr Top Biophys 2001;24:171-183.

3. Marshall KA, Reiter RJ, Poeggeler B, Aruoma OI, Halliwell B. Evaluation of the antioxidant activity of melatonin in vitro. Free Radic Biol Med 1996;21: 307-315.

4. Reiter R, Tang L, García JJ, Muñoz-Hoyos A. Pharmacological actions of melatonin in oxygen radical pathophysiology. Life Sci 1997;60:2255-2271.
5. Reiter RJ. Cytoprotective properties of melatonin: Presumed association with oxidative damage and aging. Nutrition 1998;14:691-696.

6. Reiter RJ, Calvo JR, Karbownik M, Qi W, Tan DX. Melatonin and its relation to the immune system and inflammation. Ann N Y Acad Sci 2000;917:376-386.

7. Maestroni GJ. The immunotherapeutic potential of melatonin. Expert Opin Investig Drugs 2001;10: 467-476.

8. García-Maurino S, Pozo D, Calvo JR, Guerrero JM. Correlation between nuclear melatonin receptor expression and enhanced cytokine production in human lymphocytic and monocyte cell lines. J Pineal Res 2000;29:129-137.

9. Nakade O, Koyama H, Ariji H, Yajima A, Kaku T. Melatonin stimulates proliferation and type I collagen synthesis in human bone cells in vitro. $J$ Pineal Res 1999;27:106-110.

10. Koyama H, Nakade O, Takada Y, Kaku T, Lay KH. Melatonin at pharmacologic doses increases bone mass by suppressing resorption through down-regulation of the RANKL-mediated osteoclast formation and activation. $J$ Bone Miner Res 2002;17:1219-1229.

11. Laakso ML, Porkka-Heiskanen T, Alila A, Stenberg D, Johansson NG. Correlation between salivary and serum melatonin: Dependence on serum melatonin levels. J Pineal Res 1990;9:39-50.

12. Mclntyre IM, Norman TR, Burrows GD, Armstrong SM. Melatonin rhythm in human plasma and saliva. $J$ Pineal Res 1987;4:177-183.

13. Mandel ID. Sialochemistry in diseases and clinical situations affecting salivary glands. Crit Rev Clin Lab Sci 1980;12:321-366.

14. Vakkuri O. Diurnal rhythm of melatonin in human saliva. Acta Physiol Scand 1985;124:409-412.

15. Cutando A, Gómez-Moreno G, Villalba J, Ferrera MJ, Escames G, Acuña-Castroviejo D. Relationship between salivary melatonin levels and periodontal status in diabetic patients. J Pineal Res 2003;35:239-244.

16. World Health Organization. Oral Health Surveys: Basic Methods, 4th ed. Geneva: World Health Organization; 1987:31-32.

17. Miles A, Thomas DR, Grey JE, Pugh AJ. Salivary melatonin assay in laboratory medicine longitudinal profiles of secretion in healthy men. Clin Chem 1987; 33:1957-1959.

18. Arendt J, Paunier L, Sizonenko PC. Melatonin radioimmunoassay. J Clin Endocrinol Metab 1975;40: 347-350.

19. Acuña-Castroviejo D, Escames G, Carazo A, León J, Khaldy H, Reiter RJ. Melatonin mitochondrial homeostasis and mitochondrial-related diseases. Curr Top Med Chem 2002;2:133-151.

20. Reiter RJ. Aging and oxygen toxicity: Relation to changes in melatonin. Age (Omaha) 1997;20: 201-213.

21. García-Maurino S, González-Haba MG, Calvo JR, Goberna R, Guerrero JM. Involvement of nuclear binding sites for melatonin in the regulation of IL-2 and IL-6 production by human blood mononuclear cells. J Neuroimmunol 1998;92:76-84.

22. Cardinali DP, Ladizesky MG, Boggio V, Cutrera RA, Mautalen C. Melatonin effects on bone: Experimental facts and clinical perspectives. J Pineal Res 2003; 34:81-87.

23. Boyle WJ, Simonet WS, Lacey DL. Osteoclast differentiation and activation. Nature 2003;423:337-342. 
24. Liu D, Xu J, Figliomeni L, et al. Expression of RANKL and OPG mRNA in periodontal disease: Possible involvement in bone destruction. Int J Mol Med 2003; $11: 17-21$.

25. Theoleyre S, Wittrant Y, Kwan Tat S, Fortun Y, Redini F, Heymann D. The molecular triad OPG/RANK/ RANKL: Involvement in the orchestration of pathophysiological bone remodelling. Cytokine Growth Factor Rev 2004;15:457-475.

26. Masui T, Sakano S, Hasegawa Y, Warashina H, Ishiguro $N$. Expression of inflammatory cytokines, RANKL and OPG induced by titanium, cobalt-chromium and polyethylene particles. Biomaterials 2005;26: 1695-1702.

27. Takahashi N, Nobuyuki U, Takami M, Suda T. Cells of bone. Osteoclast generation. In: Bilezikian J, Raisz L, Rodan G, eds. Principles of Bone Biology, 2nd ed. San Diego: Academic Press; 2002:109-126.

28. Sandyk R, Anastasiadis P, Anninos P, Tsagas N. Is postmenopausal osteoporosis related to pineal gland functions? Int J Neurosci 1992;62:215-225.

29. Ostrowska Z, Kos-Kudla B, Swietochowska E, Marek B, Kajdaniuk D, Gorski J. Assessment of the relationship between dynamic pattern of nighttime levels of melatonin and chosen biochemical markers of bone metabolism in a rat model of postmenopausal osteoporosis. Neuroendocrinol Lett 2001;22: 129-136.

30. Guerrero J, Pozo D, García-Mauriño S, Osuna C, Molinero P, Calvo J. Involvement of nuclear receptors in the enhanced IL-2 production by melatonin in Jurkat cells. Ann N Y Acad Sci 2000;917:397-403.

31. Väänänen K, Zhao H. Osteoclast function. Biology and mechanism. In: Bilezikian J, Raisz L, Rodan G, eds. Principles of Bone Biology, 2nd ed. San Diego: Academic Press; 2002:127-141.

32. Roth J, Kim BG, Lin WL, Cho MI. Melatonin promotes osteoblast differentiation and bone formation. J Biol Chem 1999;274:22041-22047.

33. Battino M, Bullón P, Wilson M, Newman H. Oxidative injury and inflammatory periodontal diseases: The challenge of anti-oxidants to free radicals and reactive oxygen species. Crit Rev Oral Biol Med 1999;10: 458-476.

Correspondence: Dr. Pablo Galindo, C/Recogidas 39, 5 Izda, Granada, Spain. Fax: 34-958244085; e-mail: pgalindo@ ugr.es.

Accepted for publication March 30, 2006. 